Kragujevac Journal of Mathematics

Volume 40(2) (2016), Pages 166-171.

\title{
ON A CONVEXITY PROPERTY
}

\author{
SLAVKO SIMIĆ
}

\begin{abstract}
In this article we proved an interesting property of the class of continuous convex functions. This leads to the form of pre-Hermite-Hadamard inequality which in turn admits a generalization of the famous Hermite-Hadamard inequality. Some further discussion is also given.
\end{abstract}

\section{INTRODUCTION}

Most general class of convex functions is defined by the inequality

$$
\frac{\phi(x)+\phi(y)}{2} \geq \phi\left(\frac{x+y}{2}\right) .
$$

A function which satisfies this inequality in a certain closed interval $I$ is called convex in that interval. Geometrically it means that the midpoint of any chord of the curve $y=\phi(x)$ lies above or on the curve.

Denote now by $Q$ the family of weights i.e., non-negative real numbers summing to 1. If $\phi$ is continuous, then the inequality

$$
p \phi(x)+q \phi(y) \geq \phi(p x+q y)
$$

holds for any $p, q \in Q$. Moreover, the equality sign takes place only if $x=y$ or $\phi$ is linear (cf. [1]).

The same is valid for so-called Jensen functional, defined as

$$
\partial_{\phi}(\mathbf{p}, \mathbf{x}):=\sum p_{i} \phi\left(x_{i}\right)-\phi\left(\sum p_{i} x_{i}\right)
$$

where $\mathbf{p}=\left\{p_{i}\right\}_{1}^{n} \in Q, \mathbf{x}=\left\{x_{i}\right\}_{1}^{n} \in I, n \geq 2$.

Key words and phrases. Continuous convex functions, Pre-Hermite-Hadamard inequalities, Generalization of Hermite-Hadamard inequality.

2010 Mathematics Subject Classification. 39B62(26D15).

Received: February 27, 2016.

Accepted: July 1, 2016. 
Geometrically, the inequality (1.1) asserts that each chord of the curve $y=\phi(x)$ lies above or on the curve.

\section{Results And Proofs}

Main contribution of this paper is the following

Proposition 2.1. Let $f(\cdot)$ be a continuous convex function defined on a closed interval $[a, b]:=I$. Denote

Prove that

$$
F(s, t):=f(s)+f(t)-2 f\left(\frac{s+t}{2}\right) .
$$

$$
\max _{s, t \in I} F(s, t)=F(a, b) .
$$

Proof. It suffices to prove that the inequality

$$
F(s, t) \leq F(a, b)
$$

holds for $a<s<t<b$.

In the sequel we need the assertion stated in Lemma 2.1 (which is of independent interest).

Lemma 2.1. Let $f(\cdot)$ be a continuous convex function on some interval $I \subseteq \mathbb{R}$. If $x_{1}, x_{2}, x_{3} \in I$ and $x_{1}<x_{2}<x_{3}$, then

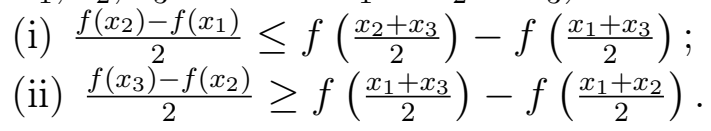

We shall prove the first part of the lemma; proof of the second part goes along the same lines.

Since $x_{1}<x_{2}<\left(x_{2}+x_{3}\right) / 2<x_{3}$, there exist $p, q ; 0 \leq p, q \leq 1, p+q=1$ such that $x_{2}=p x_{1}+q\left(\frac{x_{2}+x_{3}}{2}\right)$. Hence,

$$
\begin{aligned}
\frac{f\left(x_{1}\right)-f\left(x_{2}\right)}{2}+f\left(\frac{x_{2}+x_{3}}{2}\right) & =\frac{1}{2}\left[f\left(x_{1}\right)-f\left(p x_{1}+q \frac{x_{2}+x_{3}}{2}\right)\right]+f\left(\frac{x_{2}+x_{3}}{2}\right) \\
& \geq \frac{1}{2}\left[f\left(x_{1}\right)-\left(p f\left(x_{1}\right)+q f\left(\frac{x_{2}+x_{3}}{2}\right)\right)\right]+f\left(\frac{x_{2}+x_{3}}{2}\right) \\
& =\frac{q}{2} f\left(x_{1}\right)+\frac{2-q}{2} f\left(\frac{x_{2}+x_{3}}{2}\right) \\
& \geq f\left(\frac{q}{2} x_{1}+\frac{2-q}{2}\left(\frac{x_{2}+x_{3}}{2}\right)\right) \\
& =f\left(\frac{q}{2} x_{1}+\left(\frac{x_{2}+x_{3}}{2}\right)-\frac{1}{2}\left(x_{2}-p x_{1}\right)\right) \\
& =f\left(\frac{x_{1}+x_{3}}{2}\right) .
\end{aligned}
$$


For the proof of second part we can take $x_{2}=p\left(\frac{x_{1}+x_{2}}{2}\right)+q x_{3}$ and proceed as above.

Now, applying the part (i) with $x_{1}=a, x_{2}=s, x_{3}=b$ and the part (ii) with $x_{1}=s$, $x_{2}=t, x_{3}=b$, we get

$$
\begin{aligned}
& \frac{f(s)-f(a)}{2} \leq f\left(\frac{s+b}{2}\right)-f\left(\frac{a+b}{2}\right) ; \\
& \frac{f(b)-f(t)}{2} \geq f\left(\frac{s+b}{2}\right)-f\left(\frac{s+t}{2}\right),
\end{aligned}
$$

respectively.

Subtracting (2.2) from (2.3), the desired inequality follows.

Remark 2.1. A challenging task is to find a geometric proof of the property (2.1).

We shall quote now a couple of important consequences. The first one is used in a number of articles although we never saw a proof of it.

Corollary 2.1. Let $f$ be defined as above. If $x, y \in[a, b]$ and $x+y=a+b$, then

$$
f(x)+f(y) \leq f(a)+f(b) .
$$

Proof. Obvious, as a simple application of Proposition 2.1.

Corollary 2.2. Under the conditions of Proposition 2.1, the double inequality

$$
2 f\left(\frac{a+b}{2}\right) \leq f(p a+q b)+f(p b+q a) \leq f(a)+f(b)
$$

holds for arbitrary weights $p, q \in Q$.

Proof. Applying Proposition 2.1 with $s=p a+q b, t=p b+q a ; s, t \in I$ we get the right-hand side of (2.4). The left-hand side inequality is obvious since, by definition,

$$
\frac{f(p a+q b)+f(p b+q a)}{2} \geq f\left[\frac{(p a+q b)+(p b+q a)}{2}\right]=f\left(\frac{a+b}{2}\right) .
$$

Remark 2.2. The relation (2.4) represents a kind of pre-Hermite-Hadamard inequalities. Indeed, integrating both sides of $(2.4)$ over $p \in[0,1]$, we obtain the form of HermiteHadamard inequality (cf. [2]),

$$
f\left(\frac{a+b}{2}\right) \leq \frac{1}{b-a} \int_{a}^{b} f(t) d t \leq \frac{f(a)+f(b)}{2} .
$$

Moreover, the inequality (2.4) admits a generalization of the Hermite-Hadamard inequality.

Proposition 2.2. Let $g$ be an arbitrary non-negative and integrable function on $I$. Then, with $f$ defined as above, we get

$$
2 f\left(\frac{a+b}{2}\right) \int_{a}^{b} g(t) d t \leq \int_{a}^{b}(g(t)+g(a+b-t)) f(t) d t \leq(f(a)+f(b)) \int_{a}^{b} g(t) d t
$$


Proof. Multiplying both sides of (2.4) with $g(p a+q b)$ and integrating over $p \in[0,1]$, we obtain

$$
2 f\left(\frac{a+b}{2}\right) \frac{\int_{a}^{b} g(t) d t}{b-a} \leq \frac{\int_{a}^{b}(f(t)+f(a+b-t)) g(t) d t}{b-a} \leq(f(a)+f(b)) \frac{\int_{a}^{b} f(t) d t}{b-a},
$$

and, because

$$
\int_{a}^{b}(f(t)+f(a+b-t)) g(t) d t=\int_{a}^{b}(g(t)+g(a+b-t)) f(t) d t
$$

the inequality (2.5) follows.

We shall give in the sequel some illustrations of this proposition.

Corollary 2.3. For any $f$ that is convex and continuous on $I:=[a, b], 0<a<b$ and $\alpha \in \mathbb{R} /\{0\}$, we have

$$
2 f\left(\frac{a+b}{2}\right) \leq \frac{\alpha}{b^{\alpha}-a^{\alpha}} \int_{a}^{b}\left[t^{\alpha-1}+(a+b-t)^{\alpha-1}\right] f(t) d t \leq f(a)+f(b) .
$$

Also, for $\alpha \rightarrow 0$, we get

\section{Corollary 2.4.}

$$
2 f\left(\frac{a+b}{2}\right) \frac{\log (b / a)}{a+b} \leq \int_{a}^{b} \frac{f(t)}{t(a+b-t)} d t \leq[f(a)+f(b)] \frac{\log (b / a)}{a+b} .
$$

Similarly,

\section{Corollary 2.5.}

$$
\begin{aligned}
& 2 f\left(\frac{\pi}{2}\right) \leq \int_{0}^{\pi} f(t) \sin t d t \leq f(0)+f(\pi) \\
& 2 f\left(\frac{\pi}{4}\right) \leq \int_{0}^{\pi / 2}[\sin t+\cos t] f(t) d t \leq f(0)+f\left(\frac{\pi}{2}\right) .
\end{aligned}
$$

Estimations of the convolution of symmetric kernel on a symmetric interval are also of interest.

Corollary 2.6. Let $f$ and $g$ be defined as above on a symmetric interval $[-a, a], a>0$. Then we have that

$$
2 f(0) \int_{-a}^{a} g(t) d t \leq \int_{-a}^{a}[g(-t)+g(t)] f(t) d t \leq[f(-a)+f(a)] \int_{-a}^{a} g(t) d t .
$$

Remark 2.3. There remains the question of possible extensions of the relation (2.1). In this sense one can try to prove, along the lines of the proof of (2.1), that

$$
\max _{p, q \in Q ; x, y \in[a, b]} F^{*}(p, q ; x, y)=F^{*}(p, q ; a, b),
$$

where

$$
F^{*}(p, q ; x, y):=p f(x)+q f(y)-f(p x+q y)
$$


Anyway the result will be wrong, as simple examples show (apart from the case $\left.f(x)=x^{2}\right)$.

On the other hand, it was proved in [3] that for $p_{i} \in Q$ and $x_{i} \in[a, b]$ there exist $p, q \in Q$ such that

$$
\partial_{f}(\mathbf{p}, \mathbf{x})=\sum p_{i} f\left(x_{i}\right)-f\left(\sum p_{i} x_{i}\right) \leq p f(a)+q f(b)-f(p a+q b),
$$

for any continuous function $f$ which is convex on $[a, b]$.

Therefore, an important conclusion follows.

Corollary 2.7. For arbitrary $p_{i} \in Q$ and $x_{i} \in[a, b]$, we have that

$$
\sum p_{i} f\left(x_{i}\right)-f\left(\sum p_{i} x_{i}\right) \leq \max _{p}[p f(a)+q f(b)-f(p a+q b)]:=T_{f}(a, b),
$$

where $T_{f}(a, b)$ is an optimal upper global bound, depending only on a and $b$ (cf. [3]).

An answer to the above remark is given by the next

Proposition 2.3. If $f$ is continuous and convex on $[a, b]$, then

$$
\max _{p, q \in Q ; x, y \in[a, b]} F^{*}(p, q ; x, y) \leq F(a, b) .
$$

Proof. We shall prove just that

$$
F^{*}(p, q ; x, y) \leq F(x, y),
$$

for all $p, q \in Q$ and $x, y \in[a, b]$.

Indeed,

$$
\begin{aligned}
F(x, y)-F^{*}(p, q ; x, y) & =q f(x)+p f(y)+f(p x+q y)-2 f\left(\frac{x+y}{2}\right) \\
& \geq f(q x+p y)+f(p x+q y)-2 f\left(\frac{x+y}{2}\right) \\
& \geq 2 f\left(\frac{(q x+p y)+(p x+q y)}{2}\right)-2 f\left(\frac{x+y}{2}\right) \\
& =0 .
\end{aligned}
$$

The rest of the proof is an application of Proposition 2.1.

Putting there $x=a, y=b$ and combining with (2.6), we obtain another global bound for Jensen functional.

Corollary 2.8. We have that

$$
\mathcal{J}_{f}(\mathbf{p}, \mathbf{x}) \leq f(a)+f(b)-2 f\left(\frac{a+b}{2}\right):=T_{f}^{\prime}(a, b) .
$$

The bound $T_{f}^{\prime}(a, b)$ is not so precise as $T_{f}(a, b)$ but is much easier to calculate. 


\section{REFERENCES}

[1] G. H. Hardy, J. E. Littlewood and G. Polya, Inequalities, Cambridge University Press, Cambridge, 1978.

[2] C. P. Nikulesku and L. E. Persson, Old and new on the Hermite-Hadamard inequality, Real Anal. Exchange 29(2) (2003/4), 663-685.

[3] S. Simić, Best possible global bounds for Jensen functional, Proc. Amer. Math. Soc. 138(7) (2010), 2457-1462.

Mathematical Institute SANU

Kneza Mihaila 36, 11000

Belgrade, SERBiA

E-mail address: ssimic@turing.mi.sanu.ac.rs 